\title{
Ballistic hole emission spectroscopy of self-assembled GeSi/Si(001) nanoislands
}

\author{
(C) D.O. Filatov, D.V. Guseinov, V.Yu. Chalkov, S.A. Denisov, V.G. Shengurov \\ Lobachevsky State University of Nizhny Novgorod, \\ 603950 Nizhny Novgorod, Russia \\ E-mail: dmitry_filatov@inbox.ru
}

Ballistic hole emission microscopy spectroscopy has been applied to study the electronic structure of the hole states in the self-assembled $\mathrm{GeSi} / \mathrm{Si}(001)$ nanoislands. The ballistic hole emission microscopic images demonstrated the spots of increased collector current related to the ballistic electron tunnelling via the confined valence band states in the $\mathrm{GeSi} / \mathrm{Si}(001)$ nanoislands. In the ballistic hole emission spectra of the Ge hut clusters the stepwise features attributed to the quantum confined hole states have been observed. The results of present study demonstrate the capabilities of the ballistic hole emission microscopy spectroscopy in the characterization of the electronic structures of the valence band states in the $\mathrm{GeSi} / \mathrm{Si}$ nanostructures.

\section{Acknowledgements}

This work has been supported by Ministry of Education and Science of Russian Federation (Project 16.16.7443.2017). The BHEM/BHES measurements have been performed using the shared research facilities of Research and Educational Center for Physics of Solid State Nanostructures at Lobachevskii State University of Nizhny Novgorod. 